\title{
How to Implement a Simulation-Based Education Programme: Lessons from the UK Urology Simulation Boot Camp
}

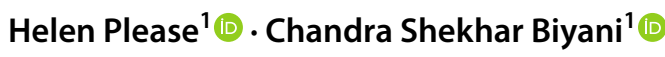

Received: 10 June 2021 / Accepted: 19 June 2021 /Published online: 7 July 2021

(c) Crown 2021

\begin{abstract}
The operative and non-technical skills exposure of urology trainees has reduced due to a number of factors, including the European Working Time Directive, and the COVID-19 pandemic. Simulation-based education (SBE) is an innovative addition to clinical experience which can begin to address the skills-based learning deficiency in order to help trainees meet their curriculum requirements and optimise the exposure required for a trainee to become a competent general urology consultant. Surgical simulation is an effective training tool but has a complex implementation process, requiring considerable planning tailored to specific educational targets, to ensure it is sustainable and reproducible. Methodology from the field of implementation science offers an invaluable approach to design an effective simulation-based training adjunct, as exemplified by the example of the UK Urology Simulation Boot Camp (USBC), a comprehensive training course which incorporates core technical and non-technical skills based on the current Joint Committee on Surgical Training (JCST) urological training curriculum to equip newly appointed urology trainees to work as competent junior registrars. Delivered annually in Leeds since 2015, the course has had excellent feedback and results in improving the urological knowledge of trainees, as well as increases in trainees' confidence. This paper will provide a summary of how the course was designed, delivered, reproduced, sustained and evaluated. Its success is demonstrated by its incorporation into the UK urology training programme, and since 2018, it is now recommended to all new urology residents in the UK. The course implementation model would be applicable to other surgical specialties.
\end{abstract}

Keywords Urology $\cdot$ Simulation $\cdot$ Implementation science $\cdot$ Education $\cdot$ Teaching $\cdot$ Surgical skills $\cdot$ Training

\section{Introduction}

Over the past few years, the shape of urological training in the UK has undergone significant changes, due to a number of important factors. Firstly, the introduction of the European Working Time Directive has altered the working hours of trainees, although the decision of the UK to leave the EU may result in further changes. Secondly, the pressures to deliver consultant-led care are increasing, which can reduce skills-based learning for juniors. Finally, and most recently, the COVID-19 pandemic has led to a significant reduction in elective operating worldwide [1]. These factors compound

Chandra Shekhar Biyani

shekharbiyani@hotmail.com

Helen Please

helen.please@nhs.net

1 Department of Urology, Saint James' Hospital, Leeds Teaching Hospitals NHS Trust, Leeds, UK the longstanding need to ensure urology trainees develop skills to a level of competency appropriate for practising as consultants at the end of their training. To this end, the registrar training posts must encompass multi-faceted demands, including adequate exposure to managing basic urological conditions, sufficient operative opportunities to develop technical skills and support to progress in non-technical proficiencies such as communication.

Urology experience is variable in the 2-year core surgical training programme and 2-year foundation training which UK-based trainees undergo prior to entering Higher Specialty Training (HST) in urology or another surgical subspecialty [2,3]. As a result, trainees starting HST in urology do not always have the adequate urological experience to equip them to be competent to the level of a day-1 urology registrar. Furthermore, even in HST, the roughly 50 UK urology trainees selected annually are spread across regions and hospitals with variable urological services. This leads to discrepancies in trainees' exposure to the urological 
subspecialties required to complete the Joint Committee on Surgical Training (JCST) Urology curriculum [4-7]. This challenge is not particular to the UK, with heterogeneity in urological training being identified across a number of European countries [8]. In summary, it is difficult to standardise operative experience to an optimal standard for all trainees, particularly in the lead up to HST. Simulated training is thought to help standardise exposure to a breadth of urological skills and decision-making necessary for safe practice as a registrar $[9,10]$.

The steep transition in responsibility and required knowledge required from a Core Surgical Training post to practising as a Urology Registrar can be daunting, particularly if a trainee has limited exposure to urology. As a result, innovative methods are required to equip trainees, and ensure adequate clinical experience. It has been estimated that the accumulated experience required in a surgeon's journey from medical school to a consultancy post (including foundation, core training and fellowships) is over 10,000 h. Not only has the ability to accumulate this clinical experience been somewhat reduced, but another barrier is that training costs have shifted towards the responsibility of the trainee. For example, study budgets are often insufficient even for mandatory courses and exams [11, 12]. Simulation is a growing field with the potential to shorten learning curves and standardise training opportunities alongside the clinical environment [13-18]. This is particularly relevant in times when clinical opportunities are rarer, such as the current COVID-19 pandemic. The UK urology curriculum contains several procedures which are amenable to simulation, exemplified by simulators designed specifically for ureteroscopy, cystoscopy, transurethral resection of the bladder (TURBT) and transurethral resection of the prostate (TURP) $[15,19-21]$. The engagement of trainees in such simulated procedures has been shown to be beneficial to improving clinical and operative skills $[9,22]$. This style of training is applicable to a range of surgical specialties; however, this paper focuses on the field of urology training.

Simulation-based education (SBE) has been shown to be an effective medical education tool; however, it is still in its infancy, and its multi-faceted design and implementation process are complex. Its success, therefore, depends on considerable planning which is tailored to specific educational targets, with much to be learnt from the field of implementation science, which will be further discussed later in this article. The training implemented must be sustainable, reproducible and ideally scalable, without overreliance on those who initially developed the programme. UK-based simulation courses for trainees often focus on 1-2 procedures over 1-2 days; however, more intensive and regular simulation may have larger benefits. We present the design and implementation of an evidence-based surgical simulation training programme for newly appointed UK
Urology registrars entitled the 'Urology Simulation Boot Camp' (USBC). This course was designed with the aim of preparing newly appointed junior registrars to start urology training in the UK, through an intensive simulation training course focussed on skill and knowledge acquisition. The 5-day course has run annually since 2015 and is tailored to the UK training syllabus, at the level required to work as a competent junior urology registrar. It compromises a 1:1 faculty-to-delegate ratio and includes modules in technical and non-technical skills (Table 1). This paper will provide a summary of how the course was developed, including how methods from implementation science have been successfully utilised to inform the design and delivery of this programme.

\section{Course Objectives}

Our aim was to introduce a comprehensive training course for newly appointed urology registrars across the UK, which incorporates core technical and non-technical skills training at a central location. The content was based on the current JSCT urological training curriculum to provide a standardised foundation of urological knowledge and skills required to work as a competent junior urology registrar. The benefits of positioning the course at the start of the registrar training scheme was thought to optimise the opportunity to practise the skills early in training, reduction of potential anxiety trainees may experience prior to starting a new role and maximisation of patient safety by ensuring trainees learn good habits as early as possible in their careers [24]. There is the additional benefit that new trainees can meet their colleagues from hospitals nationwide who they will be working alongside during their 5-year training, and future consultancy posts. This can improve teamworking skills and sharing of knowledge.

In order to achieve the objective of the intensive USBC providing a realistic reflection of skills a newly appointed registrar would require, an evidence-based approach was required to allow a structured trainee-centred design. It was recognised that the development and implementation of a simulation programme is a complex, multi-phase and demanding process. Therefore, the course lead educators chose to utilise implementation science methodologies in the programme's development, as its efficacy in healthcare simulation is broadly acknowledged $[25,26]$. This approach was intended to reduce any 'trial-and-error' tendencies, as well as to optimise the needs and expectations of those learning, delivering and ultimately receiving care from the simulated training. We adopted the well-established evidence-based Fixsen's six-stage [27, 28] implementation framework throughout the process of design and delivery, from the exploration phase to the sustainability phase of the USBC. 
Table 1 Current curriculum for the Urology Boot Camp, organised by modules
Module 1: core procedures

Module 2: reconstructive urology

Module 3: laparoscopy

Module 4: endourology 1

Module 5: scenario 1

Module 6: female/functional

Module 7: scenario 2

Module 8: endourology 2

Additional/evening sessions

Modules trialled and removed/modified (2015-2021)
- Inguino-scrotal surgery

- Scrotal examination

- Testicular fixation/hydrocele

- SPC \& catheter troubleshooting

- Priapism

- Penile fracture*

- Bowel anastomosis

- Stoma formation

- Ileal conduit diversion

- Ureteric re-implantation**

- Bladder repair**

- Basic laparoscopic skills-E-BLUS

- TURP—wet and electronic simulators

- TURBT

- Instruments*

- Bladder washout*

- Infected obstructed kidney

- Pelvic fracture/urethral injury

- Autonomic dysreflexia*

- Renal trauma

- Pneumothorax

- Urodynamics

- Mid-urethral tapes

- Botox administration

- Urethral bulking agents

- Simulated ward round/emergency scenarios*

- Communication scenarios/outpatients*

- Cystoscopy*

- Stent insertion*

- Ureteroscopy (rigid/flexible)*

- Paediatric urology**

- Talk on professionalism from $\mathrm{RCS}^{* *}$

- BUJI knowledge session**

- Quality improvement and audit**

- Circumcision, Mid-urethral tapes

- Laser and ionising radiation, GreenLight laser

- Suprapubic catheterisation (replaced by catheter troubleshooting and SPC)

- Technology and stent insertion (energy source, equipment, cystoscopy/stent, cystoscopy/washout)

- Uro-radiology

- Outpatient consultation skills and stepping up to ST3

- Medico-legal lecture, consent, capacity, leadership, Admitting when mistakes are made

- Human factors talk

- Professionalism and ISCP curriculum

- Stepping up from CT to ST

Modified and adapted from a figure by Young et al. [23]

$S P C$ suprapubic catheter, E-BLUS European training in basic laparoscopic urological skills, RCS Royal College of Surgeons

*Skills added in 2016

**Skills added in 2017
The programme development took about 18 months. Research suggests successful SBE requires a multi-disciplinary approach to design, development, delivery and evaluation of SBE learning experiences for trainees. Therefore, the input and endorsement of a wide range of stakeholders was sought. For an educational perspective, these included trainees, programme directors, the Dean, the Chair of the Specialty Advisory Committee, the Hospital Director of 
Education and the British Association of Urological Surgeons. For a clinical perspective, those included were urologists, anaesthetists, nurses, nurse consultants, acute medical physicians, simulation experts in technical and non-technical skills, information technology experts and psychologists.

\section{Course Design Considerations}

The cost of the course was considered carefully. For example, there is consideration of the potential financial impact to trainees wanting to improve their skills and clinical safety. Therefore, it was decided that the total course fee should be within the study budget available to the majority of urology registrars, in order to reduce personal financial burden to trainees. The cost of travelling to the course was also considered, resulting in the choice of a venue located centrally in the UK, in Leeds. The quality of the simulation training was prioritised through a 1:1 faculty-to-delegate training ratio and the aim of a consultant-grade faculty (quality first approach). This demand for a large number of senior urologists was potentially very expensive. This cost was avoided by inviting retired consultant urologists from across the UK to act voluntarily as faculty for course delivery, without compromising the ratio. The approach was very successful, with over 50 urology consultants volunteering as faculty, including a core group who return annually to teach on the course, as well as new trainers joining annually. Due to the intense nature of the course, extra faculty members are always planned, in order to provide well-deserved rest to faculty members between courses. In addition, extra faculty can compensate for any last-minute faculty absences. Furthermore, a number of industry sponsorship were also secured, whose financial support helped cover equipment costs, reducing overall course costs to the trainees.

The quality of the simulation training was thought to be optimal if delivered through a dedicated simulation and training suite. This led to the chosen location for programme delivery being a dedicated simulation centre in a large university teaching hospital centrally located within the UK. The initial capacity was determined to be 16 delegates at the inaugural USBC in 2015, which acted as a pilot. There was a significant growth in capacity the following year to 32 delegates, and the most recent in-person course delivery accommodated 48 delegates.

The experience from the USBC pilot course facilitated the final design of the full 5-day USBC with 8 modules delivered over 4 days (see Table 1), with an assessment on the final day. The main learning points were that the majority of the trialled course content, models and assessment methods worked well. In addition, the administrative and technical staff functioned well as a team, and support and funding from the industry partners were invaluable. Industry partners also provided positive feedback about their involvement assurance of sustained support, as well as useful feedback from trainees which allowed the identification of strengths and limitations of the pilot, to inform improvement of future course delivery.

\section{Course Content}

The course structure was designed by dividing the existing curriculum into eight modules (Table 1), with an assigned lead educator for each to allow logistical coordination of course components. As the complexity and dynamic nature of the healthcare environment increases, today's trainees are expected to possess not only specialty-specific technical skills but also a wide range of non-technical skills [29-31], both of which were incorporated into the course content. The module topics were selected by a committee of consultant urologists with an interest in surgical training, in order to cover each aspect of the JSCT urological training syllabus for the first 2 years of registrar training [4]. In addition, to the practical modules, the final course structure included a mixture of workshops and lectures to incorporate several curriculum topics which are often more difficult for trainees to get exposure to, including paediatric urology, investigating incidents and the relevance of quality improvement in urology. Once the topics and time frames of each were determined, it was evaluated that by delivering modules in half-day sessions (4 h), with workshops and lectures interspersed, the content could be intensively delivered over a 4-day period. This allowed planning for a fifth day of practical and written assessment to culminate the knowledge and provide trainees with an objective outcome.

Pre-course information was made available to each delegate prior to the course for the purposes of reducing course length, augmenting consolidation of learning and maximising 'hands-on' training. This was designed to integrate the concepts and rationale behind each procedure delivered on the course, based on the JCST Urology HST curriculum. The pre-course material was accessible to trainees via an online portal and included written and interactive video material.

In regard to practical skill teaching, we developed several 'in-house' models for the course, with positive results [32-34]. The design of the course took into account an aim that all delegates achieve an adequate amount of practice of basic urological skills which trainees would be expected to perform as registrars. A minimum objective was established: during the course, the majority of delegates should perform specific core procedures a minimum of five times. Those identified as core procedures to a junior registrar role were TURBT, TURP and several ureteroscopic procedures. In order to optimise the knowledge base alongside the practical learning, a short introductory lecture (20 to $30 \mathrm{~min}$ ) 
was incorporated into the start of the six technical modules (modules 1, 2, 3, 4, 6 and 8; Table 1). The content was a reiteration of the pre-course material related to each procedure. To ensure a consistent minimum skills outcome for all delegates, it was decided that faculty members should ensure each participant completes each simulated procedure at least once during each skills session. To allow delegates to receive feedback on their technical skills, there was an ongoing assessment of each delegate's technical ability and surgical skills throughout each of these modules.

The high delegate-to-faculty ratio permitted an unparalleled opportunity to collect evidence for each trainee from multiple expert assessments over 5 days, individualised to the trainee's competence level and progress. To formalise this feedback, following the successful completion of the course, a detailed 'trainee summary report' was compiled for each trainee and shared with them. This allows a unique sustained learning opportunity from the course, because the report can be used to guide trainees with their future training needs. This could be done in initial discussion with their Academic Educational Supervising Consultant, as the report provides an instant appreciation of the trainees' performance amongst other candidates of similar ability and career aspirations. Such information is valuable for the trainee and their supervisor while planning their initial placement goals, based on the current competency levels and training requirements.

\section{Implementation Science}

Designing successful clinical programmes for surgical trainees is only the first step; what is more difficult is the multi-dimensional process of transferring and sustaining these programmes to real-life settings. The newly emerged field of implementation science has significant potential to address this issue by optimising and standardising SBE, particularly in regard to course delivery and sustainability. Implementation science is a derivative of the hugely instrumental evidence-based medicine (EBM) movement. Implementation science is defined as the study of methods to promote the systematic uptake of evidence-based programmes and practices into a health setting and thus to improve the quality and effectiveness of intervention delivery [35]. This is a field which addresses the issues of why some robust EBM interventions fail in regard to sustainability and loss of momentum when application moves from the research setting to the clinical 'real-world'. Furthermore, the use of an implementation framework allows different stages of the implementation process to be separated into manageable components, as well as offering continuous evidence-based programme improvement in subsequent stages.
If this implementation is used correctly, it has significant potential to optimise and standardise SBE, particularly in terms of course delivery and sustainability. To the best of our knowledge, the integration of implementation science with SBE is a novel idea; therefore, we would like to demonstrate its enormous potential through the example of the USBC, which is a complex educational programme whose success was established through implementation science. Ultimately, we hope to increase accessibility to implementation concepts for clinicians and educationalists, to facilitate their application to future surgical training programmes.

There is an excellent track record of improving patient outcomes by learning from industries outside of healthcare, and studies regarding implementation science in other industries demonstrate implementation of learning is limited by "not a poor understanding of environmental forces or inappropriate strategic intent... they knew what they had to do; their difficulties lay in how to achieve the necessary changes" [36]. This is a common issue in surgical teaching, as many surgical departments have strong motivations to support trainees learning, but are limited in knowing how best to channel their efforts to achieve effective outcomes. Implementation science has been demonstrated to be successful in a growing number of disciplines, including medical education, novel technologies in healthcare and paediatric health in diverse settings [37-39]. To harness the benefits of SBE fully, we would argue that an evidence-based implementation approach is essential, and some policymakers have developed collaborative work to explore suitable methods to transform implementation evidence into practice.

There is a vast array of validated frameworks for implementation [40, 41]. Irrespective of which is used, their use provides a strategy to enable educators to plan their intervention according to the wider context, implementation need and stage of maturity. For the USBC implementation, we adopted the six stages of the implementation framework by Fixsen et al. [27, 28]. This framework is composed of the following stages of programme implementation: (1) exploration, in which the existing situation is assessed; (2) installation, in which changes are deliberated; (3) initial implementation, in which preparations are made for the changes; (4) full implementation, in which change is in process; (5) innovation, in which adaptations to the practising interventions are made to suit the user; and (6) sustainability, in which procedures are maintained. The USBC has followed implementation science principles to successfully improve delivery, sustainability and innovations. Its success is demonstrated by its incorporation into the UK urology training programme, and being recommended to all new urology residents in the UK from 2018. The key principles of the course, from inception to adaptations are as follows: ' 1 trainee-1 trainer-1 model' template, maximum hands-on experience, adherence and adaptation to the national training 
curriculum, equal weight to technical and non-technical skills and course delivery at the start of the urology training programme.

In terms of replication and sustainability, once well established, the course model was used to reproduce similar courses in Belgium (2018-2019), Portugal (2018-2019) and Zambia (2019). The adaptability of the course to different settings requires modification to the environment, in particular consideration of available resources and educational needs of the trainees. For each location, the course was tailored to suit the needs of first-year urology residents in that country, while maintaining the important fabric of the USBC carried out annually in Leeds, UK. In these novel settings, a modified 1-day pilot course was delivered, with excellent feedback. Consequently, the European School of Urology has adopted the model and included a boot camp as a first step for the newly appointed urology trainee. Recently in April 2021, the model was successfully used to deliver the first obstetrics and gynaecology boot camp in Leeds.

\section{Course Assessment}

Any novel educational programme requires rigorous evaluation and improvement. Therefore, in order to assess the usefulness of the USBC, we performed sequential assessments of delegates at several critical time points: prior to the USBC, immediately following the USBC and 3 months following the USBC. These online assessments incorporated subjective and objective markers through multiple-choice questions (MCQs) and Likert scale questionnaires. By using the same assessment at each timepoint, it was possible to assess how much knowledge, confidence and skills were gained through the course, and whether this learning was maintained in the months beyond the USBC, prior to and following the course. These assessments allowed quantitative comparison of each delegate's level of urological knowledge, operative competency and operative confidence. The knowledge included general urological knowledge and selfassessed confidence in operative and non-technical skills (such as leading a urology ward round).

In addition to assessments filled out by delegates, the experienced faculty also assessed each delegates' technical skill progression throughout the course. The final day of the course was particularly important for this, with an assessment of technical skills in core urological procedures; the five selected were TURP, endoscopic instrument assembly, E-BLUS (the European training in basic laparoscopic urological skills) laparoscopic skills, female pelvic examination and urodynamics and rigid ureteroscopy. Every delegate was evaluated on each skill module, both at the time of teaching and on the final assessment day using a validated instrument. They were given a score according to a grading system from
1 (poor) to 5 (excellent, the level expected for a certificate of completion of training). These were determined by a consultant faculty member who was blinded to individual scores in the modular training, and participants were unaware of which skills would be assessed.

\section{Evaluation of Outcomes}

The USBC started a pilot-scale national course with 16 delegates (2015) and, over 3 years, has successfully grown to accommodate 48 delegates (2017). It has been funded and incorporated into the UK national training curriculum as a 'mandatory' course for newly appointed urology registrars, as well as being recommended by the Education Committee of the British Association of Urological Surgeons and the JCST. Furthermore, the course has been adapted to be successfully delivered in a diversity of high-income and lowand middle-income settings.

On evaluation of the assessment results and feedback from delegates and faculty, the USBC has been shown to have a very positive impact on training. Feedback from delegates and faculty members over the years of course delivery has been overwhelmingly positive. In terms of delegate perception for the 2015, 2016 and 2017 courses, when asked if the course met the trainees learning objectives and provided necessary knowledge and skills, the majority selected 'strongly agreeing' (55.9-93.75\%). The level of urological knowledge was shown to improve, in all years for the 2015, 2016 and 2017 courses. This was demonstrated by a mean examination score across these years of 55.5\% prior to the course and $70.1 \%$ following the course. In terms of the delegates' self-assessed confidence in performing core urological procedures, this was shown to significantly improved immediately after the course in all five technical skills assessed (analysed using Student's $t$ test) and was sustained for most procedures at 3 months after the course [23]. Those procedures which did not show a sustained improvement in confidence at 3 months were those less frequently performed by trainees (i.e. mid-urethral tape insertion), which is to be expected. Overall, the improvement in technical ability demonstrated through intensive simulation training is consistent with the evidence in contemporary literature that both high- and low-fidelity surgical simulators are useful tools in the acquisition of core urological skills $[15,19-21]$. For further details on how an intensive surgical simulation course can improve competency progression, our previous reports on evaluating trainee progression during simulation training highlight the issue [24]. Delegate feedback regarding non-technical skills demonstrated that delegates felt able to further their skills in communication, delegation and prioritisation. The validated behaviour rating system 'Non-Technical Skills for Surgeons' (NOTSS) [42] 
was used to analyse the non-technical modules of the course ( 5 and 7 , see Table 1), allowing the reflective practice, which was well received by delegates.

Overall, we feel these outcomes demonstrate that the USBC offers an effective and realistic simulated training programme for new urology registrars which accurately and comprehensively covers the JSCT syllabus. Having such a course placed as an accessible or even mandatory requirement at the start of a new registrar position prepares trainees to put their newly acquired knowledge and skills into practice from day 1 of the job. This optimises training opportunities and patient safety, reduces anxiety and aids planning ongoing training needs. The 'Trainee Summary Report' helps trainees and their supervisors relate their performance to other trainees at their stage. This facilitates the onward planning of clinical training goals at supervisor meetings.

\section{Course Adaptations}

The extensive feedback collected from delegates and faculty throughout the course delivery and development cannot be over-emphasised. Critical feedback allows adaptation of course delivery annually to enhance learning and trainee satisfaction. It is well established in implementation science that a dynamic, ongoing evaluation of a programme is required to identify strengths and limitations and improve it to continue to have sustainable delivery. Constructive criticism was used to make meaningful alterations to improve the course for training needs. Examples include early course feedback which highlighted that the circumcision and suprapubic catheterisation content, delivered in the 2015 course, were skills which trainees usually attain in Core Surgical Training prior to their registrar appointment in urology. Delegates, therefore, expressed a preference for teaching on catheter troubleshooting rather than insertion, which was incorporated into the 2016 and 2017 courses with positive effect. Another example was that the early courses included evening lectures; however, the feedback demonstrated that delegates felt these were not effective as they were tired following $8 \mathrm{~h}$ of simulation training in the day. Therefore, the learning content originally delivered in the evening lectures was instead incorporated into interactive discussions in the morning and workshops for the 2017 course, with positive feedback.

Throughout the years of course delivery, regular critical evaluation of feedback has been carried out amongst the core course organising group. A full report is regularly compiled and shared with sponsors, and results have been continually published in peer-reviewed journals [23, 24, 43]. In addition, many key stakeholders have been invited to observe the course delivery, with the aim of encouraging faculty members and allowing sponsors to see the immediate impact of their support. As a result of these regular and transparent updates with our supporters, we have successfully secured funding for the course every year.

We accept that there are limitations in our assessment of confidence, knowledge and technical ability as these were established on the last day of the course, at a time when it might be expected that knowledge retention is optimal. Although we tried to negate this by re-assessing 3 months after the course, there may be confounding factors such as clinical and operative experience during the delegate's training placement. These confounding factors are difficult to remove from any analysis.

\section{Conclusion}

Overall, the success of the USBC highlights the enormous potential for surgical training offered by incorporating implementation strategies into developing SBE courses. The design, delivery and sustainability of an effective simulation programme is an immense undertaking, requiring commitment from a multi-disciplinary array of stakeholders. This implementation process can be considered scientifically as a sequential series of interconnected steps which should be adopted to maximise the potential quality and success of any educational intervention. We strongly advocate the use of the implementation science in the design of any SBE strategy. The financial implications for those running and attending any course require careful thought. The sustainability of SBE is essential, requiring rigorous feedback and assessment to inform adaptations of the course design and delivery. Replicability is also important, and there are challenges of successfully transferring such a programme from one setting to another. This requires a holistic approach that takes into account the context-specific variables to adapt a SBE programme successfully. Consequently, the principles of implementation science should be maintained to maximise the benefits of every simulation programme, whether local or national. Our example of the success of the USBC nationally, and internationally, clearly demonstrates that implementation science has significant scope in SBE design and delivery. We implore other surgical educationalists to adopt implementation methodology for future innovations in order to successfully identify barriers, reduce resource wastage and gain maximal acceptance from stokeholds, good return of faculty and satisfied competent trainees, and ultimately, we hope this approach improves patient outcomes.

Acknowledgements We would like to thank all participants and the entire faculty for their support and developing the Urology Simulation Boot Camp. 
Author Contribution All authors contributed to the study conception and design. Material preparation, data collection and analysis were performed by Chandra Shekhar Biyani. The first draft of the manuscript was written by Helen Please, and all authors commented on previous versions of the manuscript. All authors read and approved the final manuscript.

Funding There was no financial or other support for the writing of this article. There were local and industry sponsors who support the running of the course which the article is based on, referenced in the manuscript:

1. Equipment sponsorship provided by Karl Storz, Cook Medical, Coloplast, Ethicon, Dantec, OKB Medical/Simbionix, MediPlus, Teleflex and European Pharma

2. Teaching facilities accommodated by Medical Education Leeds and Leeds Teaching Hospitals NHS Trust

Data Availability Available on request from the authors.

Code Availability Not applicable.

\section{Declarations}

Ethics Approval and Consent to Participate Not applicable.

Consent for Publication Not applicable.

Competing Interests The authors declare no competing interests.

\section{References}

1. Hope C, Reilly JJ, Griffiths G, Lund J, Humes D (2021) The impact of COVID-19 on surgical training: a systematic review. Tech Coloproctol 25(5):505-520

2. Bolton JF, Lemberger RJ, Neligan P, Eardley I (2009) National selection and recruitment in urology: 2009. Br J Med Surg Urol 2(6):225-232

3. Lyttle M, Jones R, Mukherjee R (2017) Urology-are we leaving junior doctors behind? MedEdPublish, A prospective study, p 6

4. Cornford P, Foster L, Hodgson D, Morley R, Lane T, Parkinson R (2021) Urology curriculum, the Intercollegiate Surgical Curriculum Programme. General Medical Council. https://www. gmc-uk.org/education/standards-guidance-and-curricula/curri cula/urology-curriculum. Accessed 28 Apr 2021

5. Robinson R, O'Flynn KJ (2015) Indicative operative numbers in urology training in the UK and Ireland. J Clin Urol 8(3):188-195

6. Gill JD, Stewart LF, George NJ, Eardley I (2012) Operative experience of urological trainees in the UK. BJU Int 109(9):1296-1301

7. Cleaveland P, Jones C, Thompson A, Liew M, Husain J (2017) Exposure to paediatric urology during urology specialty training: a UK national trainee survey. Bull R Coll Surg Engl 99(9):332-335

8. Carrion DM, Gómez Rivas J, Esperto F, Patruno G, Vasquez JL (2018) Current status of urological training in Europe. Arch Esp Urol 71(1):11-17

9. Wignall GR, Denstedt JD, Preminger GM, Cadeddu JA, Pearle MS, Sweet RM, McDougall E (2008) Surgical simulation: a urological perspective. J Urol 179(5):1690-1699
10. Canalichio KL, Berrondo C, Lendvay TS (2020) Simulation training in urology: state of the art and future directions. Adv Med Educ Pract 11:391

11. Harrison E, Shalhoub J (2007) The cost of surgical training: position statement by the Association of Surgeons in Training. The Association of Surgeons, London (in Training)

12. Giddings $C$ (2011) The effects of rising costs of surgical training. Association of Surgeons in Training, London

13. Bjerrum F, Thomsen ASS, Nayahangan LJ, Konge L (2018) Surgical simulation: current practices and future perspectives for technical skills training. Med Teach 40(7):668-675

14. Ramirez AG, Hu Y, Kim H, Rasmussen SK (2018) Long-term skills retention following a randomized prospective trial on adaptive procedural training. J Surg Educ 75(6):1589-1597

15. Neumann E, Mayer J, Russo GI, Amend B, Rausch S, Deininger S, Bedke J et al (2019) Transurethral resection of bladder tumors: next-generation virtual reality training for surgeons. Eur Urol Focus 5(5):906-911

16. Somasundram K, Spence H, Colquhoun AJ, Mcilhenny C, Biyani CS, Jain S (2018) Simulation in urology to train nontechnical skills in ward rounds. BJU Int 122(4):705-712

17. Veneziano D, Cacciamani G, Biyani CS (2018) Simulation and training in urology-in collaboration with ESU/ESUT. Arch Esp Urol 71(1):55-62

18. Aggarwal G, Adhikary SD (2017) Simulators in the urological training armamentarium: a boon or a bane? Arab J Urol 15(2):166-169

19. Viswaroop SB, Gopalakrishnan G, Kandasami SV (2015) Role of transurethral resection of the prostate simulators for training in transurethral surgery. Curr Opin Urol 25(2):153-157

20. He ZH, Zhang Y, Liu JS, Gang WA, Yu CF, Na YQ (2013) Virtual reality simulator for training urologists on transurethral prostatectomy. Chin Med J 126(7):1220-1223

21. Hu WG, Feng JY, Wang J, Song YJ, Xu XT, Zhou H, Huang CB (2015) Ureteroscopy and cystoscopy training: comparison between transparent and non-transparent simulators. BMC Med Educ 15(1): 1-9

22. Chou DS, Abdelshehid C, Clayman RV, McDougall EM (2006) Comparison of results of virtual-reality simulator and training model for basic ureteroscopy training. J Endourol 20(4):266-271

23. Young M, Kailavasan M, Taylor J, Cornford P, Colquhoun A, Rochester M, Jain S et al (2019) The success and evolution of a urological "boot camp" for newly appointed UK urology registrars: incorporating simulation, nontechnical skills and assessment. J Surg Educ 76(5):1425-1432

24. Kailavasan M, Hanchanale V, Rajpal S, Morley R, Mcllhenny C, Somani B, Myatt A et al (2019) A method to evaluate trainee progression during simulation training at the Urology Simulation Boot Camp (USBC) course. J Surg Educ 76(1):215-222

25. Price DW, Wagner DP, Krane NK, Rougas SC, Lowitt NR, Offodile RS et al (2015) What are the implications of implementation science for medical education? Med Educ Online 20:27003

26. Haji FA, Da Silva C, Daigle DT, Dubrowski A (2014) From bricks to buildings: adapting the Medical Research Council framework to develop programs of research in simulation education and training for the health professions. Simul Healthc 9(4):249-259

27. Fixsen DL, Blase KA, Naoom SF, Wallace F (2009) Core implementation components. Res Soc Work Pract 19(5):531-540

28. Fixsen DL, Naoom SF, Blase KA, Friedman RM, Wallace F, Burns B, Shern D et al (2005) Core implementation components. In Implementation research: a synthesis of the literature. Tampa, FL: University of South Florida, Louis de la Parte Florida Mental Health Institute, The National Implementation Research Network FMHI Publication 231(4):23-34 
29. Aditya I, Kwong JC, Canil T, Lee JY, Goldenberg MG (2020) Current educational interventions for improving technical skills of urology trainees in endourological procedures: a systematic review. J Endourol 34(7):723-731

30. Hull L, Sevdalis N (2015) Advances in teaching and assessing nontechnical skills. Surgical Clinics 95(4):869-884

31. Yamane M, Sugimoto S, Suzuki E, Aokage K, Okazaki M, Soh J, Toyooka S et al (2020) Continuing surgical education of nontechnical skills. Ann Med Surg 58:177-186

32. Berridge CT, Kailavasan M, Logan M, Johnson J, Biyani CS, Taylor J (2021) A training model to teach early management of priapism. Actas Urol Esp (Engl Ed) 45(3):220-224

33. Bratt DG, Berridge C, Young M, Kailavasan M, Taylor J, Biyani CS (2020) A simple novel training model for teaching suprapubic catheter (SPC) exchange. Actas Urol Esp (Engl Ed) 44(8):549-553

34. Kailavasan M, Berridge C, Kandaswamy G, Rai B, Wilkinson B, Jain S, Gowda B et al (2020) A low-cost synthetic abdominal wall model ("Raj Model") for the training of laparoscopic port insertion. World J Surg 44(5):1431-1435

35. Eccles MP, Mittman BS (2006) Welcome to implementation science. Implementation Sci 1:1. https://doi.org/10.1186/ 1748-5908-1-1

36. Bartlett CA, Ghoshal S (1987) Managing across borders: new organizational responses. Sloan Management Review 29(1):43-53

37. Price DW, Wagner DP, Krane NK, Rougas SC, Lowitt NR, Offodile RS, Barnes BE et al (2015) What are the implications of implementation science for medical education? Med Educ Online 20(1):27003
38. Harris C, Garrubba M, Allen K, King R, Kelly C, Thiagarajan M, Farjou D et al (2015) Development, implementation and evaluation of an evidence-based program for introduction of new health technologies and clinical practices in a local healthcare setting. BMC Health Serv Res 15(1):1-16

39. Westgard C, Fleming WO (2020) The use of implementation science tools to design, implement, and monitor a community-based mHealth intervention for child health in the Amazon. Front Public Health 8:411

40. Moullin JC, Sabater-Hernández D, Fernandez-Llimos F, Benrimoj SI (2015) A systematic review of implementation frameworks of innovations in healthcare and resulting generic implementation framework. Health Res Pol Syst 13(1):1-11

41. Tabak RG, Khoong EC, Chambers DA, Brownson RC (2012) Bridging research and practice: models for dissemination and implementation research. Am J Prev Med 43(3):337-350

42. Yule S, Flin R, Maran N, Youngson G, Mitchell A, Rowley D, Paterson-Brown S (2008) Debriefing surgeons on non-technical skills (NOTSS). Cogn Technol Work 10(4):265-274

43. Hanchanale V, Kailavasan M, Rajpal S, Koenig P, Yiasemidou M, Palit V, Biyani CS et al (2019) Impact of urology simulation boot camp in improving endoscopic instrument knowledge. BMJ Simul Technol Enhanc Learn 5(3):151-154

Publisher's Note Springer Nature remains neutral with regard to jurisdictional claims in published maps and institutional affiliations. 